\title{
Application of Laminated Composite Grids as a Reinforcing Element of Automotive Components
}

\author{
Amir Ehsani \& Hamid Dalir
}

\begin{abstract}
This paper intends to present the application of laminated grid structures as a new class of stiffeners for reinforcing body and chassis of transportation vehicles. A laminated grid plate is constituted from several grid plies with different orientations. Therefore, the grid layers with various fibers, patterns, and orientations can be used, resulting in laminates with enhanced stiffness and coupling effects. In this study, a hypothetical trunk floor is assumed as a sandwich panel with two skins and a composite laminated grid core, which is clamped along all edges. Three different grid structures are considered as the core to strengthen the trunk floor subjected to arbitrary lateral loads. Moreover, the first natural frequency of the plates are achieved. The Ritz method is employed to obtain the maximum deflection and free vibration frequencies of the trunk's floor panel. The results indicate that employing the laminated grids considerably enhances the response of the panel in comparison with conventional grids.
\end{abstract}

\section{INTRODUCTION}

Due to low weight and high strength and stiffness, the grid or lattice stiffeners are widely employed in various engineering structures including marine, vehicles, and buildings. Increasing interest in composite materials and because of their low-cost automated manufacturing procedure, the composite materials are known as a good option for producing the grid structures.

Up to now, most studies are focused on modeling, prediction of mechanical behavior, and fabrication of the grid structures. Gurdal et al. [1] evaluated the structural efficiency of geodesically stiffened shells with various stiffener arrangements under compression, torsion and combined loads. Similarly, in an optimization process, Oliveira et al. [2] presented a practical method of finding a minimum weight of grid plates subjected to a lateral load. Kidane et al. [3] used the smeared method to analysis buckling of the grid stiffened shell, and validated the results with experimental results. Ambur et al. [4] developed an optimal design strategy for grid structures with variable

Amir Ehsani, Hamid Dalir, Department of Mechanical and Energy Engineering, Purdue School of Engineering and Technology, IUPUI, U.S.A. 
curvatures for global and local buckling analysis. Moreover, Chen et al. [5] presented spline compound stripe method to analysis the free vibration of a stiffened plate. Their results were in good agreement with the experimental and numerical results. Ehsani and Rezaeepazhand [6] presented a new class of grid structures which are known as "laminated grid structures". They investigated lamination effects on stiffness and mechanical behavior of grid structures. Ehsani et al. [7, 8] studied the influence of the stacking sequence and pattern composition on buckling load of laminated composite grid plates and also conducted studies on the buckling load and natural vibration of laminated orthogrid plates. Their results showed that, there are proper stacking sequences, which considerably improves the mechanical behaviors of the grid structures.

Despite the many studies on different types of grid structures there have been rare investigations on the applications of laminated grid structures. Similar to a laminated composite, laminated grid is composed of different grid layers with different orientations, which can improve the stiffness and coupling effects of conventional grids [8]. Tailored stiffness of a structure is achieved by employing full anisotropic properties of a laminated grid compare to orthotropic properties with at most in plane anisotropic of a corresponding conventional grid [6]. In current study, the laminated grids are consisting of orthogrid layers in which the principal grid directions are not necessarily parallel to the plate axes. As an example, Figure 1 illustrates two types of orthogrid structures. Figure 1(a) shows a laminated orthogrid plate, which is, composed of three orthogrid layers, with $\left(90^{\circ} / 0^{\circ} / 90^{\circ}\right)$ stacking sequence. Figure 1(b) illustrates a conventional orthogrid plate with identical thickness to the laminated orthogrid.

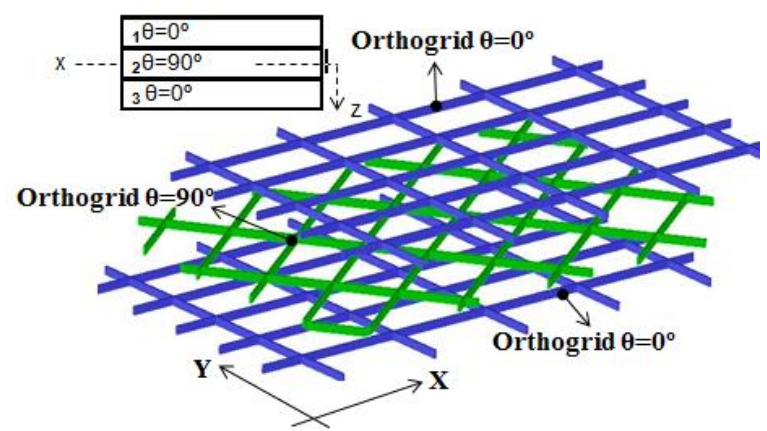

(a)

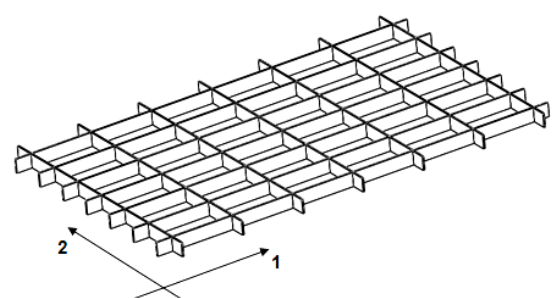

(b)

Figure 1. A Laminated orthogrid with $\left(0^{\circ} / 90^{\circ} / 0^{\circ}\right)$ configuration (a) a conventional Orthogrid plate with equal total thickness (b)

In automotive industry, many parts are susceptible to bending, deformation and vibration such as floor pan, trunk floor, doors and truck's container floor. Grid structures are one of the optimal and effective stiffeners, which are used to reinforce load-bearing components. Automotive companies always try to increase load bearing along with reduce vehicles' weight. Consequently, using composite materials are rising in car industry to decrease the chassis weight. In the present work, the laminated grid structures are presented as a feasible alternative for conventional grid elements, which can tolerate more loads with identical weight. Here, a hypothetical trunk floor is assumed as a sandwich panel with two skins and a composite grid core, which is 
clamped along all edges. Figure 2 shows a schematic view of the hypothetical trunk floor with conventional grid stiffener.

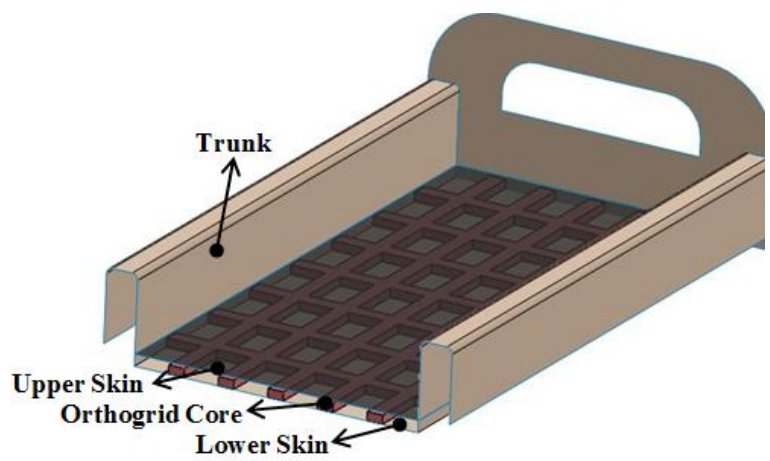

Figure 2. Schematic view of the hypothetical trunk floor with conventional orthogrid stiffener core

To investigate the advantages of laminated grid structures over conventional grids in automotive applications three different symmetric angle ply grid structures are considered. Using laminated plate theory along with Ritz method, the deflection and first natural frequency of the grid core are calculated.

\section{PROBLEM DESCRIPTION}

\section{Laminated Grid Configuration}

A laminated grid composed of several grid layers which the pattern, thickness and orientation of each layer can be varied [8]. In the current work, the laminated grids are composed of several composite orthogrid layers. Three cases of grid structures have been considered in this study. The several geometry parameters namely, $a, d_{1}, d_{2}, t_{1}, t_{2}$, are identical for conventional orthogrid and the orthogrid layers of the laminated grid plates (see Figure 3) and the thickness of the layers are defined to have identical thickness in all cases. The plates have square shape with side a, and symmetrical arrangement respect to mid-plane. The structures are considered to have clamped boundary condition along all edges.

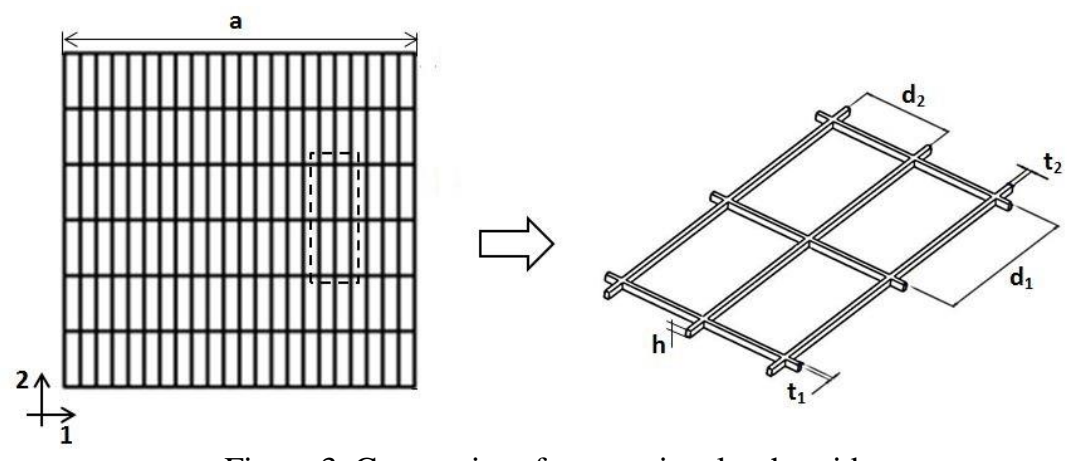

Figure 3. Geometries of conventional orthogrid 
The first case is considered to be a sub-laminate grid structure with $\left( \pm \theta^{\circ}\right) 5$ s configuration and twenty layers (see TABLE 1). The second case is a sub-laminate grid structure with $\left( \pm \theta^{\circ}\right)_{\mathrm{s}}$ configuration and four layers. The third one is a single layer orthogrid or conventional orthogrid. It is assumed that the grid layers are without any initial imperfection or defect and are perfectly bonded to each other. TABLE 1 presents the characteristics of the defined cases.

In a laminated grid, each grid layer may have any arbitrary orientation $(\theta)$. Figure 4 illustrates a special orthogrid and a general orthogrid plate, which is rotated $\theta$ degree with respect to $\mathrm{X}$ direction.
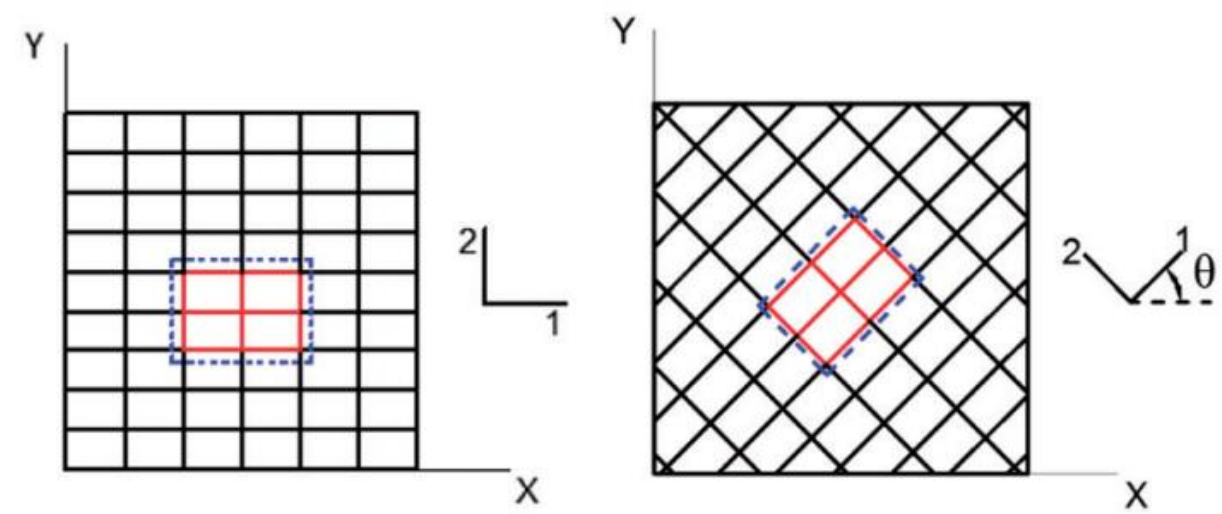

Figure 4. A specially orthogrid $(\theta=0)$ (left). A general orthogrid that is rotated $\theta$ degree with respect to $\mathrm{x}$ direction (right). [8]

The grid layers are made of carbon/epoxy material with the following properties: $E_{1}=48$ $\mathrm{GPa}, \mathrm{E}_{2}=15.3 \mathrm{GPa}, \mathrm{G}_{12}=5.1 \mathrm{GPa}, v_{12}=0.315$, and density $=2112 \mathrm{~kg} / \mathrm{m}^{3}$ [9]; where $\mathrm{E}_{1}, \mathrm{E}_{2}$, and $\mathrm{G}_{12}$ are the longitudinal, transvers elastic and shear modules of the applied material, respectively.

TABLE 1. THE CHARACTERISTICS OF THE CONSIDERED CASES

\begin{tabular}{|c|c|c|c|c|c|c|}
\hline Case \# & $\begin{array}{c}\text { Type of grid } \\
\text { structure }\end{array}$ & Lay-up name & $\begin{array}{l}\text { Stacking } \\
\text { sequence }\end{array}$ & $\begin{array}{l}\text { Number of } \\
\text { layers }(\mathrm{N})\end{array}$ & $\begin{array}{c}\text { Total } \\
\text { thickness }\end{array}$ & $\begin{array}{c}\text { Layers' } \\
\text { thickness (h) }\end{array}$ \\
\hline 1 & Laminated Orthogrid & Sub-laminate & $\left( \pm \theta^{\circ}\right)_{5 \mathrm{~s}}$ & 20 & $\mathrm{H}$ & $\mathrm{H} / 20$ \\
\hline 2 & Laminated Orthogrid & Sub-laminate & $\left( \pm \theta^{\circ}\right)_{\mathrm{s}}$ & 4 & $\mathrm{H}$ & $\mathrm{H} / 4$ \\
\hline 3 & $\begin{array}{c}\text { Conventional } \\
\text { Orthogrid }\end{array}$ & Orthogrid & $\left(\theta^{\circ}\right)$ & 1 & $\mathrm{H}$ & $\mathrm{H}$ \\
\hline
\end{tabular}

\section{CONSTITUTIVE EQUATIONS}

Similar to laminated composites grid structures have directional properties. Therefore, to obtain mechanical behaviors of a grid structure the stiffness matrices of grid ply should be calculated. The extensional, coupling and bending stiffness matrices cab be achieved according to Equation 1 [10]. 


$$
\left.\left.\left.A_{i j}, B_{i j}, D_{i j}={ }_{k=1}^{N}\left(\bar{Q}_{i j}\right)_{k} \quad z_{k}-z_{k-1}\right), \frac{1}{2} z_{k}^{2}-z_{k-1}^{2}\right), \frac{1}{3} z_{k}^{3}-z_{k-1}^{3}\right] i, j=1,2,6
$$

Where, $\mathrm{k}$ is the layer number in the laminate, $\mathrm{z}_{\mathrm{k}}$ is distance of $\mathrm{k}$ layer from the middle surface, and $\mathrm{N}$ is the number of the grid layers. In this study, it is assumed the grid structure are symmetric; therefore, the coupling stiffness matrix [B] will be equal to zero.

To achieve the stiffness matrices and consequently the deformation of grid structures the transformed reduced stiffness matrices, of each grid layer is applied according to the method which is presented by Nemeth [11]. Equation 2 presents the reduced stiffness matrix, [Q], of a conventional orthogrid layer.

$$
[Q]=\left[\begin{array}{ccc}
\frac{E_{1} t_{1}}{d_{1}} & 0 & 0 \\
0 & \frac{E_{1} t_{2}}{d_{2}} & 0 \\
0 & 0 & \frac{G_{12} t_{1}}{d_{1}}+\frac{G_{12} t_{2}}{d_{2}}
\end{array}\right]
$$

Where $d_{1}, d_{2}, t_{1}, t_{2}$ and $h$ are geometric parameters of the grid layer as illustrated in Figure 3. $E_{1}$ and $G_{12}$ are the longitudinal elastic and shear modulus of unidirectional composite materials of each layer. The transformed reduced stiffness matrix $[\bar{Q}]$ of the orthogrid layer is achieved by substituting [Q] from Equation 2 into Equation 3.

$$
[\bar{Q}]=[T]^{-1}[Q \llbracket T]^{-T}
$$

where, $[\mathrm{T}]$ is the transformation matrix [10] which is only depended on each grid layer orientation, $\theta$.

To examine the advantages of using laminated grid plates over conventional grid plates, the bending and vibrational behavior of the defined cases are compared. The Ritz method along with classical laminated plate theory are employed to obtain the maximum deflection and first natural frequency of the plates.

The total potential energy of the symmetric angle ply laminated grid plate subjected to lateral load can be expressed by the following equation [13]:

$$
\pi=\Omega+U
$$

Where, $U$ is the strain energy of bending and can be obtained from Equation 5, and $\Omega$ is the potential of external forces.

$$
\begin{aligned}
& U=\frac{1}{2} \int_{0}^{a} \int_{0}^{a}\left[D_{11}\left(\frac{\partial^{2} w}{\partial x^{2}}\right)^{2}+2 D_{12} \frac{\partial^{2} w}{\partial x^{2}} \frac{\partial^{2} w}{\partial y^{2}}+D_{22}\left(\frac{\partial^{2} w}{\partial y^{2}}\right)^{2}+4 D_{16} \frac{\partial^{2} w}{\partial x^{2}} \frac{\partial^{2} w}{\partial x \partial y}+4 D_{26} \frac{\partial^{2} w}{\partial y^{2}} \frac{\partial^{2} w}{\partial x \partial y}\right. \\
& \left.+4 D_{66}\left(\frac{\partial^{2} w}{\partial x \partial y}\right)^{2}\right] d x d y
\end{aligned}
$$


For a lateral load $\mathrm{p}$ (per unit area) the potential of external force is given by

$$
\Omega=\int_{0}^{a} \int_{0}^{a}[p w] d x d y
$$

The following trigonometric function satisfies the geometric boundary conditions,

$$
\mathrm{w}(x, y)=\sum_{\mathrm{m}=1}^{\mathrm{M}} \sum_{\mathrm{n}=1}^{\mathrm{N}} W_{m n} X_{m}(x) Y_{n}(y)
$$

Where the $\mathrm{W}_{\mathrm{mn}}$ are arbitrary unknown coefficients. The $\mathrm{X}_{\mathrm{m}}(\mathrm{x})$, and $\mathrm{Y}_{\mathrm{n}}(\mathrm{y})$ are algebraic functions that satisfy the geometrical boundary conditions and for clamped boundary condition can be defined as follows [12]:

$$
\begin{aligned}
X_{m}(x) & =\left(\frac{x}{a}\right)^{m+1}-2\left(\frac{x}{a}\right)^{m+2}+\left(\frac{x}{a}\right)^{m+3} \\
Y_{n}(y) & =\left(\frac{y}{a}\right)^{n+1}-2\left(\frac{y}{a}\right)^{n+2}+\left(\frac{y}{a}\right)^{n+3}
\end{aligned}
$$

Substituting $\mathrm{w}(\mathrm{x}, \mathrm{y})$ from Equation 7 into Equations $(5,6)$ and minimizing total energy from Equation 4 with respect to the unknown $\mathrm{W}_{\mathrm{mn}}$ coefficients, $\mathrm{M} \times \mathrm{N}$ linear and simultaneous equations will be produced:

$$
\frac{\partial(\Omega+U)}{W_{m n}}=0
$$

$\mathrm{W}_{\mathrm{mn}}$ are calculated by solving these simultaneous equations.

The energy function of the vibration of a plate can be expressed as [13]:

$$
\pi=U-T_{\max }
$$

Where $T_{\max }$ is the maximum kinetic energy of the plate, is given by Liew [13]. A minimization process of Equation 10 with respect to $\mathrm{W}_{\mathrm{mn}}$ constants, yields to an eigenvalue equation in the following form:

$$
\left(K-\omega^{2} M\right) \tilde{d}=0
$$

Where $\mathrm{M}$ is consistent mass matrix and $\omega$ is the natural frequency. In this section, the analytical solution for calculating maximum deflection and natural frequencies of the clamped grid plates are expressed. In the next section, the extracted results are presented for defined cases. 


\section{RESULTS AND DISCUSSIONS}

Figure 5 depicts the non-dimensional maximum deflection, $\overline{\mathrm{W}}_{\max }$, for orthogrid and two sub-laminate grid cases subjected to a uniform lateral load.

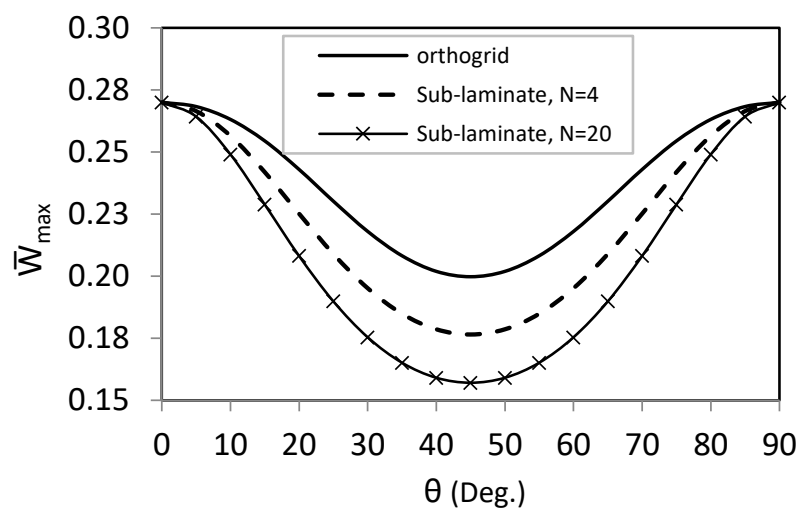

Figure 5. Non-dimensional maximum deflection of defined plates subjected to a uniform lateral load

The maximum non-dimensional deflection is defined as $\bar{W}_{\max }=\left(W_{\max } E_{1} H\right) /\left(p a^{4}\right)$, where $\mathrm{H}$ is the plates' thickness, $\mathrm{p}$ is the applied load and a, is the side of the plate. The grid plates have equal weight and dimensions. To evaluate the effects of layer orientations, $\theta$, on maximum deflection, the layer orientation has been varied from $0^{\circ}$ to $90^{\circ}$. As the figure illustrates, at $\theta=45^{\circ}$, all grid structures reach to their minimum deflection. The maximum deflection of sub-laminate grid plate with $( \pm \theta)_{\mathrm{s}}$ and $( \pm \theta)_{5 \mathrm{~s}}$ configurations are about $11 \%$ and $25 \%$ less than conventional orthogrid plate at $\theta=45^{\circ}$, respectively. As can be seen, utilizing a laminated grid, $( \pm \theta)_{5 s}$, yields to significant reduction in maximum deflection in comparison to the orthogrid with identical geometry and weight.

Figure 6 shows the non-dimensional first natural frequencies for two sub-laminated grids $\left(( \pm \theta)_{\mathrm{s}}\right.$ and $\left.( \pm \theta)_{5 \mathrm{~s}}\right)$ and conventional orthogrid plates for different layer orientations, $\theta$. Where, $\bar{\omega}=\left(\omega \mathrm{a}^{2} / \pi^{2}\right) \sqrt{\rho / D_{11}}$, and $\rho$ is the mass per unit area and $D_{11}$ is the element of bending stiffness matrix of grid plates at $\theta=0^{\circ}$.

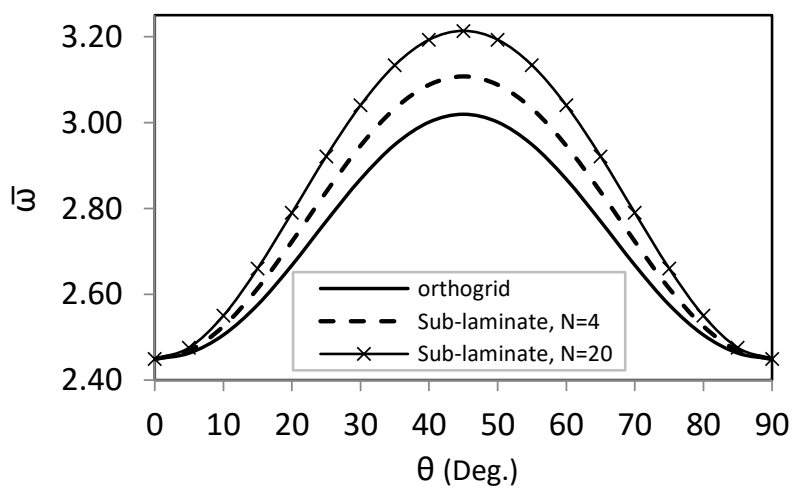

Figure 6. Non-dimensional first natural frequency for different grid orientations, $\theta$, sub-laminated grids $\left(( \pm \theta)_{\mathrm{s}}\right.$ and $\left.( \pm \theta)_{5 \mathrm{~s}}\right)$ and orthogrid plates 
According to Figure 6, similar to lateral deformation (see Figure 5), at $\theta=45^{\circ}$ the sublaminate grids and orthogrid plate achieve their maximum natural frequencies. At $\theta=45^{\circ}$ the frequency of the first case is $6.5 \%$ more than general orthogrid plate with identical orientation and is about $31 \%$ higher than corresponding specially orthogrid plate $(\theta=0)$.

\section{CONCLUSION}

In this study, the lateral deformation and first natural frequency of laminated and conventional orthogrid plates with similar weight and thickness are investigated. The results determine the considerable mechanical behavior advantages of laminated grids over conventional grids. Moreover, the results indicated that the grid orientation is the effective parameter in mechanical responses of the grid structures. In all cases at $\theta=45^{\circ}$, lateral deformation and first natural frequency obtain their best values. Furthermore, the mechanical responses are related to number of grid layers of laminated grids. Increasing the number of grid layers enhances the mechanical behaviors of the laminated grids. As result, employing a thoughtful selection of laminated grid structures can enhance the mechanical behavior of different automotive parts along with reducing the weight.

\section{REFERENCES}

1. Gürdal, Z. and G. Gendron, Optimal design of geodesically stiffened composite cylindrical shells. Composites Engineering, 1993. 3: p. 1131-1147.

2. Oliveira, J.G. and D.A. Christopoulos, A practical method for the minimum weight design of stiffened plates under uniform lateral pressure. Computers \& Structures, 1981. 14: p. 409-421.

3. Kidane, S., et al., Buckling load analysis of grid stiffened composite cylinders. Composites Part B: Engineering, 2003. 34: p. 1-9.

4. Ambur, D.R. and N. Jaunky, Optimal design of grid-stiffened panels and shells with variable curvature. Composite Structures, 2001. 52: p. 173-180.

5. Chen, C.J., W. Liu, and S.M. Chern, Vibration analysis of stiffened plates. Computers \& Structures, 1994. 50: p. 471-480.

6. Ehsani, A. and J. Rezaeepazhand, Comparison of Stiffness and Failure Behavior of the Laminated Grid and Orthogrid Plates. Journal of Solid Mechanics, 2017: p. 126-137.

7. Ehsani, A. and J. Rezaeepazhand, Stacking sequence optimization of laminated composite grid plates for maximum buckling load using genetic algorithm. International Journal of Mechanical Sciences, 2016. 119: p. 97-106.

8. Ehsani, A. and J. Rezaeepazhand, Vibration and stability of laminated composite orthogrid plates. Journal of Reinforced Plastics and Composites, 2016. 35: p. 1051-1061.

9. Naik, N.K., Y. Chandra Sekher, and S. Meduri, Damage in woven-fabric composites subjected to low-velocity impact. Composites Science and Technology, 2000. 60: p. 731-744.

10. Kollar, L.P. and G.S. Springer, Mechanics of Composite Structures. 1 ed. 2003: Cambridge University Press.

11. Nemeth, M.P., A Treatise on Equivalent-Plate Stiffnesses for Stiffened Laminated-Composite Plates and Plate-Like Lattices. 2011, NASA TP 2011-216882

12. Reddy, J.N., Mechanics of Laminated Composite Plates and Shells: Theory and Analysis. 2 ed. 2003: CRC Press.

13. Liew, K.M., Solving the Vibration of Thick Symmetric Laminates by Reissner/Mindlin Plate Theory and the p-Ritz Method. Journal of Sound and Vibration, 1996. 198(3): p. 343-360. 